\title{
RANDOM FIXED POINTS AND APPROXIMATIONS IN RANDOM CONVEX METRIC SPACES ${ }^{1}$
}

\author{
ISMAT BEG and NASEER SHAHZAD ${ }^{2}$ \\ Quaid-i-Azam University \\ Department of Mathematics \\ Islamabad, PAKISTAN
}

\begin{abstract}
Some random fixed point theorems in random convex metric spaces are obtained. Results regarding random best approximation on random convex metric spaces are also proved.
\end{abstract} metric space.

Key words: Random fixed point, random approximation,

AMS (MOS) subject classifications: 47H10, 47H04, 60H25, $41 \mathrm{~A} 50,54 \mathrm{H} 25$.

\section{INTRODUCTION AND PRELIMINARIES}

Random fixed point theory has received much attention for the last two decades, since the publication of the paper by Bharucha-Reid [3]. On the other hand, random best approximation has recently received further attention after the papers by Sehgal and Waters [13], Sehgal and Singh [12], Papageorgiou [11], Lin [10] and Beg and Shahzad [1]. The purpose of this paper is to prove some invariant random approximation theorems in random convex metric spaces.

Let $(\Omega, \mathcal{A})$ be a measurable space, $(X, d)$ a metric space, $2^{x}$ family of all subsets of $X, C K(X)$ family of all nonempty compact convex subsets of $X$, $K(X)$ family of all nonempty compact subsets of $X$ and $C B(X)$ family of all nonempty closed bounded subsets of $X$. A mapping $T: \Omega \rightarrow 2^{x}$ is called measurable if for any open subset $C$ of $X, T^{-1}(C)=\{\omega \in \Omega: T(\omega) \cap C \neq \phi\} \in \mathcal{A}$. A mapping $\xi: \Omega \rightarrow X$ is said to be a measurable selector of a measurable mapping $T: \Omega \rightarrow 2^{x}$ if $\xi$ is measurable and for any $\omega \in \Omega, \xi(\omega) \in T(\omega)$. A mapping

\footnotetext{
${ }^{1}$ Received: February, 1993. Revised: June, 1993.

${ }^{2}$ Research supported by NSRDB grant No. M.Sc. (5)/QAU/90.
} 
$f: \Omega \times X \rightarrow X$ is called a random operator if for any $x \in X, f(\cdot, x)$ is measurable. A mapping $T: \Omega \times X \rightarrow C B(X)$ is a random multivalued operator if for any $x \in X$, $T(\cdot, x)$ is measurable. A measurable mapping $\xi: \Omega \rightarrow X$ is called a random fixed point of a random multivalued (single valued) operator $T: \Omega \times X \rightarrow C B(X)$ $(f: \Omega \times X \rightarrow X)$ if for every $\omega \in \Omega, \quad \xi(\omega) \in T(\omega, \xi(\omega))(\xi(\omega)=f(\omega, \xi(\omega)))$. A mapping $T: X \rightarrow C B(X)$ is upper (lower) semicontinuous if for any closed (open) subset $C$ of $X, T^{-1}(C)$ is closed (open). A mapping $T$ is called continuous if $T$ is both upper and lower semicontinuous. A random operator $T: \Omega \times X \rightarrow C B(X)$ is called Lipschitzian if $H(T(\omega, x), T(\omega, y)) \leq L(\omega) d(x, y)$ for any $x, y \in X$ and $\omega \in \Omega$, where $L: \Omega \rightarrow[0, \infty)$ is a measurable map and $H$ is the Hausdorff metric on $C B(X)$, induced by the metric $d$. When $L(\omega)<1(L(\omega)=1)$ for each $\omega \in \Omega$, then $T$ is called contraction (nonexpansive). Let $K$ be a subset of $X$. A random operator $T: \Omega \times K \rightarrow K$ is said to be a Banach operator if there exists a measurable map $\beta: \Omega \rightarrow[0,1)$ and for each $x \in K$ and $\omega \in \Omega, d(T(\omega, T(\omega, x))$, $T(\omega, x)) \leq \beta(\omega) d(T(\omega, x), x)$. A continuous mapping $V: X \times X \times[0,1) \rightarrow X$ is said to be convex structure on $X$, if for all $x, y$ in $X$ and $\lambda \in[0,1]$ the following condition is satisfied:

$$
d(u, V(x, y, \lambda)) \leq \lambda d(u, x)+(1-\lambda) d(u, y)
$$

for all $u \in X$. A metric space $X$ with convex structure is called a convex metric space. Banach space and each of its convex subsets are simple examples of convex metric space. There are many convex metric spaces which can not be imbedded in any Banach space. For examples and other details we refer to Takahashi [14]. A subset $K$ of a convex metric space $X$ is said to be convex if $V(x, y, \lambda) \in K$ for all $x, y \in K$ and $\lambda \in[0,1]$. The set $K$ is said to be starshaped if there exists some $p \in K$ such that $V(x, p, \lambda) \in K$ for all $x \in K$ and $\lambda \in[0,1]$. The point is called starcentre of $K$. Clearly starshaped subsets of $X$ contain all convex subsets of $X$ as a proper subclass. A convex metric space is said to satisfy property $(I)$, if for all $x, y \in X$ and $\lambda \in[0,1]$,

$$
d(V(x, p, \lambda), V(y, p, \lambda)) \leq \lambda d(x, y) .
$$

Property $(I)$ is always satisfied in any normed space $X$. For details we refer to Guay, Singh and Whitfield [4]. Let $W: \Omega \times X \times X \times[0,1] \rightarrow X$ be a mapping having the following properties: 
(i) For each $\omega \in \Omega, W(\omega, \cdot, \cdot, \cdot)$ is a convex structure on $X$,

(ii) For each $x, y, \in X, \lambda \in[0,1], W(\cdot, x, y, \lambda)$ is measurable.

The mapping $W$ is called a random convex structure on $X$ and $X$ is random convex metric space.

\section{RANDOM FIXED POINTS}

In this section, random fixed point theorems in random convex metric spaces are proved. For corresponding fixed point theorems, we refer to Guay, Singh and Whitfield [4] and Beg, Shahzad and Iqbal [2].

Theorem 2.1. Let $E$ be a closed subset of a separable complete metric space $X$. Let $T: \Omega \times E \rightarrow E$ be a continuous Banach operator, then $T$ has a random fixed point.

Proof: Let $\xi_{0}: \Omega \rightarrow E$ be a measurable mapping. It follows by induction for each $\omega \in \Omega$,

$$
d\left(T^{n+1}\left(\omega, \xi_{0}(\omega)\right), T^{n}\left(\omega, \xi_{0}(\omega)\right)\right) \leq \beta^{n}(\omega) d\left(\xi_{0}(\omega), T\left(\omega, \xi_{0}(\omega)\right)\right) .
$$

Put $\xi_{1}(\omega)=T\left(\omega, \xi_{0}(\omega)\right)$. The mapping $\xi_{1}$ is measurable by Himmelberg [6] and a sequence of measurable mappings can be defined as follows:

$$
\xi_{n}(\omega)=T\left(\omega, \xi_{n-1}(\omega)\right)=T^{n}\left(\omega, \xi_{0}(\omega)\right) \quad(\text { for each } \omega \in \Omega, \quad n=1,2, \ldots) .
$$

Assuming $n \leq m$, we have for any $\omega \in \Omega$,

$$
\begin{gathered}
d\left(\xi_{n}(\omega), \xi_{m}(\omega)\right)=d\left(T^{n}\left(\omega, \xi_{0}(\omega)\right), T^{m}\left(\omega, \xi_{0}(\omega)\right)\right) \\
\leq \beta^{n}(\omega) d\left(\xi_{0}(\omega), T\left(\omega, \xi_{m-n-1}(\omega)\right)\right. \\
\leq \beta^{n}(\omega)\left[d\left(\xi_{0}(\omega), \xi_{1}(\omega)\right)+d\left(\xi_{1}(\omega), \xi_{2}(\omega)\right)+\ldots+d\left(\xi_{m-n-1}(\omega), \xi_{m-n}(\omega)\right)\right] \\
<\frac{\beta^{n}(\omega)}{1-\beta(\omega)} d\left(\xi_{0}(\omega), \xi_{1}(\omega)\right) .
\end{gathered}
$$

Since $0<\beta(\omega)<1$ for each $\omega \in \Omega,\left\{\xi_{0}(\omega)\right\}$ is a Cauchy sequence in $E$. Since $E$ being a closed subset of a complete metric space, is complete, therefore $\left\{\xi_{n}(\omega)\right\}$ converges to some $\xi(\omega) \in E$. Thus $\lim _{n \rightarrow \infty} \xi_{n}(\omega)=\xi(\omega)$. By continuity of $T$, $T(\omega, \xi(\omega))=\xi(\omega)$ for each $\omega \in \Omega$. 
Remark 2.2. Theorem 2.1 remains true if $E$ is a closed subset of a separable metric space $X$ and closure of $T(\omega, E)$ is compact for each $\omega \in \Omega$.

Theorem 2.3. Let $X$ be a separable random convex metric space satisfying property $(I)$ and $E$ be a closed and p-starshaped subset of $X$. If $T: \Omega \times E \rightarrow E$ is a nonexpansive random operator and closure of $T(\omega, E)$ is compact for each $\omega \in \Omega$, then $T$ has a random fixed point.

Proof: Define a sequence of random operators $T_{n}: \Omega \times E \rightarrow E$ by $T_{n}(\omega, x)=W\left(\omega, T(\omega, x), p, \beta_{n}(\omega)\right)$, where $\beta_{n}$ is a fixed sequence of measurable mappings $\beta_{n}: \Omega \rightarrow(0,1)$ and $\beta_{n}(\omega)$ converging to 1 . Each $T_{n}$ is a continuous Banach operator:

$$
\begin{gathered}
d\left(T_{n}(\omega, x), T_{n}^{2}(\omega, x)\right)=d\left(W\left(\omega, T(\omega, x), p, \beta_{n}(\omega)\right), W\left(\omega, T\left(\omega, T_{n}(\omega, x)\right), p, \beta_{n}(\omega)\right)\right) \\
\leq \beta_{n}(\omega) d\left(T(\omega, x), T\left(\omega, T_{n}(\omega, x)\right)\right) \\
\leq \beta_{n}(\omega) d\left(x, T_{n}(\omega, x)\right)
\end{gathered}
$$

for each $x \in E$ and $\omega \in \Omega$.

Since closure of $T(\omega, E)$ is compact, closure of $T_{n}(\omega, E)$ is compact too for each $\omega \in \Omega$, and Remark 2.2, further implies: for each $T_{n}$ there exists a random fixed point $\xi_{n}$ such that for any $\omega \in \Omega$,

$$
\xi_{n}(\omega)=T_{n}\left(\omega, \xi_{n}(\omega)\right)=W\left(\omega, T\left(\omega, \xi_{n}(\omega)\right), p, \beta_{n}(\omega)\right) .
$$

For each $n$, define $G_{n}: \Omega \rightarrow K(E)$ by $G_{n}(\omega)=c l\left\{\xi_{i}(\omega): i \geq n\right\}$. Define $G: \Omega \rightarrow K(E)$ by $G(\omega)=\bigcap_{n=1}^{\infty} G_{n}(\omega)$. Then $G$ is measurable by Himmelberg [6, Theorem 4.1] and by Kuratowski and Ryll-Nardzewski [9] has a measurable selector $\xi$. As closure of $T_{n}(\omega, E)$ is compact for each $\omega \in \Omega,\left\{\xi_{n}(\omega)\right\}$ has a subsequence $\left\{\xi_{n_{j}}(\omega)\right\}$ converging to $\xi(\omega)$. By continuity of $T$ and $W, T\left(\omega, \xi_{n_{j}}(\omega)\right)$ converges to $T(\omega, \xi(\omega))$. Thus $T(\omega, \xi(\omega))=\xi(\omega)$ for each $\omega \in \Omega$.

Theorem 2.4. Let $X$ be a compact starshaped subset of a separable random convex metric space satisfying property $(I)$. Let $T: \Omega \times X \rightarrow X$ be a nonexpansive random operator. Then $T$ has a random fixed point.

Proof: Choose a starcentre $x_{0}$ of $X$ and a sequence $\left\{k_{n}\right\}$ of measurable mappings $k_{n}: \Omega \rightarrow(0,1)$ and $k_{n}(\omega) \rightarrow 1$ as $n \rightarrow \infty$. Define the random operator $T_{n}: \Omega \times X \rightarrow X$ by 


$$
T_{n}(\omega, x)=W\left(\omega, T(\omega, x), x_{0}, k_{n}(\omega)\right) .
$$

The operator $T_{n}$ is a contraction. Indeed,

$$
\begin{gathered}
d\left(T_{n}(\omega, x), T_{n}(\omega, y)\right)=d\left(W\left(\omega, T(\omega, x), x_{0}, k_{n}(\omega)\right), W\left(\omega, T(\omega, y), x_{0}, k_{n}(\omega)\right)\right) \\
\leq k_{n}(\omega) d(T(\omega, x), T(\omega, y)) \\
\leq k_{n}(\omega) d(x, y)
\end{gathered}
$$

for all $x, y \in X$ and $\omega \in \Omega$. By Hans [5], $T_{n}$ has a unique random fixed point $\xi_{n}$. Define a sequence of mappings $G_{n}: \Omega \rightarrow K(X)$ and a mapping $G: \Omega \rightarrow K(X)$ by the same way as in the proof of Theorem 2.3. Then $G$ is measurable and has a measurable selector $\xi$. This $\xi$ is a random fixed point of $T$.

Theorem 2.5. Let $X$ be a compact and starshaped subset of a separable random convex metric space satisfying property $(I)$. Let $F: \Omega \times X \rightarrow C K(X)$ be a nonexpansive random operator, then $F$ has a random fixed point.

Proof: Choose a starcentre $p$ of $X$ and a sequence $\left\{k_{n}\right\}$ of real valued measurable mapping such that $0<k_{n}(\omega)<1$ and $k_{n}(\omega) \rightarrow 1$ as $n \rightarrow \infty$. For each $n$, defined contraction random operator, $F_{n}: \Omega \times X \rightarrow C K(X)$ by $F_{n}(\omega, x)=W\left(\omega, F(\omega, x), p, k_{n}(\omega)\right)$, then by Itoh [7] $F_{n}$ has a random fixed point $\xi_{n}$. For each $n$, define $G_{n}: \Omega \rightarrow K(X)$ by

$$
G_{n}(\omega)=\operatorname{cl}\left\{\xi_{i}(\omega): i \geq n\right\} .
$$

Define $G: \Omega \rightarrow K(X)$ by $G(\omega)=\bigcap_{n=1}^{\infty} G_{n}(\omega)$. The mapping $G$ is measurable by

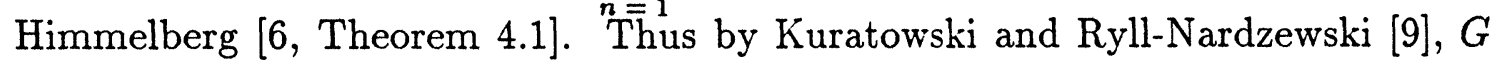
has a measurable selector $\xi$. This selector $\xi$ is the desired random fixed point of $F$. For each $n$, there exists $y_{n} \in F\left(\omega, \xi_{n}(\omega)\right)$ such that $\xi_{n}(\omega)=W\left(\omega, y_{n}, p, k_{n}(\omega)\right)$. It implies that $\left\{y_{n}\right\}$ converges to $\xi(\omega)$ and since $F$ is continuous, it follows that $\xi(\omega) \in F(\omega, \xi(\omega))$ for each $\omega \in \Omega$.

Let $(X, d)$ be a metric space. A random operator $f: \Omega \times X \rightarrow X$ is called asymptotically regular if for any $x \in X$ and $\omega \in \Omega, d\left(f^{n}(\omega, x), f^{n+1}(\omega, x)\right) \rightarrow 0$ as $n \rightarrow \infty$. A mapping $f: X \rightarrow X$ is said to commute with a mapping $F: X \rightarrow C B(X)$ if for each $x \in X, f(F(x)) \subset F(f(x))$. Also, a random operator is said to commute with a random operator $F: \Omega \times X \rightarrow C B(X)$ if for each $\omega \in \Omega, f(\omega, \cdot)$ and $F(\omega, \cdot)$ commute. 
Theorem 2.6. Let $X$ be a compact starshaped subset of a separable random convex metric space satisfying property $(I)$. Let $f: \Omega \times X \rightarrow X$ be a nonexpansive and asymptotically regular random operator, $F: \Omega \times X \rightarrow C K(X)$ be a nonexpansive random operator. Suppose $f$ commutes with $F$, then there exists a common random fixed point of $f$ and $F$.

Proof: By Theorem 2.5, $F$ has a random fixed point $\xi_{1}$. The mapping $\xi_{2}: \Omega \rightarrow X$ defined by $\xi_{2}(\omega)=f\left(\omega, \xi_{1}(\omega)\right)$ is measurable by Himmelberg [6]. Since $f$ and $F$ commute, $\xi_{2}$ is a random fixed point of $F$. By induction, the sequence $\left\{\xi_{n}\right\}$ of mapping $\xi_{n}: \Omega \rightarrow X$ for which $\xi_{n+1}(\omega)=f\left(\omega, \xi_{n}(\omega)\right) \quad(\omega \in \Omega, n=1,2, \ldots)$ are random fixed points of $F$. Define a sequence of mappings $G_{n}: \Omega \rightarrow K(X)$ and a mapping $G: \Omega \rightarrow K(X)$ by the same way as in the proof of Theorem 2.5. Then $G$ is measurable and has a measurable selector $\xi$. This $\xi$ is common random fixed point of $f$ and $F$.

Remark 2.7. With the notion of random convex metric space, our Theorems 2.5 and 2.6 generalize Theorems 3.4 and 3.6 of Itoh [8].

\section{RANDOM BEST APPROXIMATIONS}

The aim of this section is to prove some results regarding best approximation in random convex metric spaces.

A continuous function $S$ from a closed convex subset $C$ of a convex metric space $X$, into itself is said to be affine if $S(V(x, y, \lambda))=V(S x, S y, \lambda)$ whenever $\lambda \in[0,1] \cap Q$ and $x, y$ in $C$, where $Q$ denotes, the set of rational numbers. Let $(X, d)$ be a metric space and $C$ be a nonempty subset of $X$. Suppose $x \in X$. An element $y \in C$ is called an element of best approximation of $x$ (by the elements of the set $C$ ) if we have

$$
d(x, y)=\inf _{z \in C} d(x, z)
$$

We will denote by $P_{c}(x)$ the set of best $C$-approximations to $x$, that is, $P_{c}(x)=\left\{y \in C: d(x, y)=\inf _{z \in C} d(x, z)\right\}$ and boundary of $C$ by $\partial C$.

Theorem 3.1. Let $X$ be a separable random convex metric space satisfying condition $(I)$. Let $T, S: \Omega \times X \rightarrow X$ be two random operators, $C$ a subset of $X$ such that $T(\omega, \cdot): \partial C \rightarrow C$ and $x^{*}=T\left(\omega, x^{*}\right)=S\left(\omega, x^{*}\right)$ for each $\omega \in \Omega$. Further 


$$
\begin{gathered}
d(T(\omega, x), T(\omega, y)) \leq d(S(\omega, x), S(\omega, y)), \text { and } \\
d(S(\omega, x), S(\omega, y)) \\
\leq \mu(\omega) \max \left\{d(x, y), d(x, S(\omega, x)), d(y, S(\omega, y)), \frac{d(x, S(\omega, y)+d(y, S(\omega, x))}{2}\right\}
\end{gathered}
$$

[where $\mu: \Omega \rightarrow[0,1)$ is a measurable map]

for all $x, y \in P_{c}\left(x^{*}\right) \cup\left\{x^{*}\right\}$ and $\omega \in \Omega$. Let $S$ be continuous and affine on $P_{c}\left(x^{*}\right)$ and $S(\omega, T(\omega, x))=T(\omega, S(\omega, x))$ for all $x \in P_{c}\left(x^{*}\right)$. If $P_{c}\left(x^{*}\right)$ is nonempty, compact, and q-starshaped with respect to $q=S(\omega, q)$ and $S\left(\omega, P_{c}\left(x^{*}\right)\right)=P_{c}\left(x^{*}\right)$, then there exists a measurable map $\xi: \Omega \rightarrow P_{c}\left(x^{*}\right)$ which is a common random fixed point of $T$ and $S$.

Proof: If $y \in P_{c}\left(x^{*}\right)$, then for any $\omega \in \Omega$

$$
\begin{gathered}
d\left(T(\omega, y), x^{*}\right)=d\left(T(\omega, y), T\left(\omega, x^{*}\right)\right) \\
\leq d\left(S(\omega, y), S\left(\omega, x^{*}\right)\right) \\
=d\left(S(\omega, y), x^{*}\right) .
\end{gathered}
$$

Now using $S\left(\omega, P_{c}\left(x^{*}\right)\right)=P_{c}\left(x^{*}\right)$, we obtain that $T(\omega, y) \in P_{c}\left(x^{*}\right)$ for each $\omega \in \Omega$.

Let $\left\{k_{n}\right\}$ be a sequence of measurable mappings $k_{n}: \Omega \rightarrow(0,1)$ and $k_{n}(\omega) \rightarrow 1$ as $n \rightarrow \infty$. Define a random operator

$$
T_{n}: \Omega \times P_{c}\left(x^{*}\right) \rightarrow P_{c}\left(x^{*}\right)
$$

as $T_{n}(\omega, x)=W\left(\omega, T(\omega, x), q, k_{n}(\omega)\right)$. Since $S$ is affine and commutes with $T$ on $P_{c}\left(x^{*}\right)$, we have for each $\omega \in \Omega$,

$$
\begin{gathered}
T_{n}(\omega, S(\omega, x))=W\left(\omega, T(\omega, S(\omega, x)), S(\omega, q), k_{n}(\omega)\right) \\
=W\left(\omega, S(\omega, T(\omega, x)), S(\omega, q), k_{n}(\omega)\right) \\
=S\left(\omega, W\left(\omega, T(\omega, x), q, k_{n}(\omega)\right)\right. \\
=S\left(\omega, T_{n}(\omega, x)\right) .
\end{gathered}
$$

Thus $S$ commutes with $T_{n}$ on $P_{c}\left(x^{*}\right)$ for each $n$ and $T_{n}\left(\omega, P_{c}\left(x^{*}\right)\right) \subseteq P_{c}\left(x^{*}\right)=S\left(\omega, P_{c}\left(x^{*}\right)\right)$ for each $\omega \in \Omega$. Furthermore for any $\omega \in \Omega$ and $x, y \in P_{c}\left(x^{*}\right)$, 


$$
\begin{gathered}
d\left(T_{n}(\omega, x), T_{n}(\omega, y)\right) \leq k_{n}(\omega) d(T(\omega, x), T(\omega, y)) \\
\leq k_{n}(\omega) d(S(\omega, x), S(\omega, y))
\end{gathered}
$$

By Beg and Shahzad [1, Theorem 3.2] there exists a measurable map $\xi_{n}(\omega)$ $=T_{n}\left(\omega, \xi_{n}(\omega)\right)=S\left(\omega, \xi_{n}(\omega)\right)$ for each $\omega \in \Omega$. Define a sequence of mappings $G_{n}: \Omega \rightarrow K\left(P_{c}\left(x^{*}\right)\right)$ and a mapping $G: \Omega \rightarrow K\left(P_{c}\left(x^{*}\right)\right)$ by the same way as in the proof of Theorem 2.3. Then $G$ is measurable and has a measurable selector $\xi$. Since $P_{c}\left(x^{*}\right)$ is compact, $\left\{\xi_{n}(\omega)\right\}$ has a subsequence $\xi_{n_{j}}(\omega) \rightarrow \xi(\omega)$. Now $\xi_{n_{j}}(\omega)=T_{n_{j}}\left(\omega, \xi_{n_{j}}(\omega)\right)$. Since $k_{n_{j}}(\omega) \rightarrow 1$, therefore $\xi_{n_{j}}(\omega) \rightarrow T(\omega, \xi(\omega))$. Hence $T(\omega, \xi(\omega))=\xi(\omega)$ for each $\omega \in \Omega$. The continuity of $S$ further implies that

$$
S(\omega, \xi(\omega))=S\left(\omega, \lim _{n \rightarrow \infty} \xi_{n_{j}}(\omega)\right)=\lim _{n \rightarrow \infty} \xi_{n_{j}}(\omega)=\xi(\omega)
$$

for all $\omega \in \Omega$.

A metric space $X$ is called $\eta$-chainable if for every $a, b \in X$, there exists an $\eta$-chain that is a finite set of points $a=x_{0}<x_{1}<x_{2}<\ldots<x_{n}=b \quad(n$ may depend on both $a$ and $b)$ such that $d\left(x_{i-1}, x_{i}\right)<\eta \quad(i=1,2, \ldots, n)$.

A random operator $T: \Omega \times X \rightarrow X$ is said to be locally contractive if for every $x \in X$ there exists $\epsilon>0$ and a measurable map $\lambda: \Omega \rightarrow[0,1)$ which may depend on $x$ such that $p, q \in S(x, \epsilon)=\{y \in X: d(x, y)<\epsilon\}$ implies that $d(T(\omega, p), T(\omega, q) \leq \lambda(\omega) d(p, q)$ for all $\omega \in \Omega$. A random operator $T$ is called $(\epsilon, \lambda(\omega))$-uniformly locally contractive if it is locally contractive and both $\epsilon$ and $\lambda: \Omega \rightarrow[0,1)$ do not depend on $x$.

Theorem 3.2. Let $X$ be a separable random convex $\eta$-chainable metric space satisfying $(I)$ and $T: \Omega \times X \rightarrow X$ be a random operator. Let $C$ be a $T(\omega, \cdot)$ invariant subset of $X$ and $x^{*}$ be a $T(\omega, \cdot)$-invariant point for each $\omega \in \Omega$. If $P_{c}\left(x^{*}\right)$ is nonempty, compact and $p$-starshaped and $T$ is

(i) continuous on $P_{c}\left(x^{*}\right)$,

(ii) $\quad d(x, y) \leq d\left(x^{*}, C\right) \quad$ implies $\quad d(T(\omega, x), T(\omega, y))<d(x, y) \quad$ for $\quad$ all $x, y \in P_{c}\left(x^{*}\right)$ and $\omega \in \Omega$.

Then there exists a measurable map $\xi: \Omega \rightarrow P_{c}\left(x^{*}\right)$ such that for any $\omega \in \Omega$ $T(\omega, \xi(\omega))=\xi(\omega)$.

Proof: Similar to the proof of Theorem 3.1, only need to notice that the corresponding $T_{n}$ are uniformly locally contractive random operators and 
have random fixed points $\xi_{n}$ from Beg and Shahzad [2, Theorem 5.1].

We recall that an operator $T: X \rightarrow X$ is compact, if for any bounded subset $S$ of $X, C 1(T(S))$ ic compact. If an operator $T: X \rightarrow X$ leaves subset $Y$ of $X$ invariant, then a restriction of $T$ to $Y$ will be denoted by the symbol $T / Y$.

Theorem 3.3. Let $X$ be a separable random convex metric space satisfying property $(I)$ and $T: \Omega \times X \rightarrow X$ be a nonexpansive random operator. Let $C$ be a $T(\omega, \cdot)$-invariant subset of $X$ and $T(\omega, \cdot) / C$ be compact and $x^{*}$ be a $T(\omega, \cdot)$-invariant point for each $\omega \in \Omega$. If $P_{c}\left(x^{*}\right)$ is nonempty, convex and compact, then there exists a measurable map $\xi: \Omega \rightarrow P_{c}\left(x^{*}\right)$ such that $T(\omega, \xi(\omega))=\xi(\omega)$ for each $\omega \in \Omega$.

Proof: The set $P_{c}\left(x^{*}\right)$ is $T(\omega, \cdot)$-invariant, closed and convex. Since $P_{c}\left(x^{*}\right)$ is bounded subset of $c$ and $T(\omega, \cdot) / C$ is compact, closure of $\left(T\left(\omega, P_{c}\left(x^{*}\right)\right)\right)$ is compact for each $\omega \in \Omega$. Theorem 2.3 implies that $T$ has a random fixed point $\xi$ in $P_{c}\left(x^{*}\right)$.

\section{REFERENCES}

[1] Beg, I., Shahzad, N., Random fixed points of random multivalued operators on Polish spaces, Nonlinear Anal. 20 (7) (1993), 835-847.

[2] Beg, I., Shahzad, N., Iqbal, M., Fixed point theorems and best approximation in convex metric spaces, Approx. Theory and its Appl. 8 (4) (1992), 97-105.

[3] Bharucha-Reid, A.T., Fixed point theorems in probabilistic analysis, Bull. Amer. Math. Soc. 82 (1976), 641-657.

[4] Guay, M.D., Singh, K.L., Whitfield, J.H.M., Fixed point theorems for nonexpansive mappings in convex metric spaces, Proc. Conf. on Nonlinear Analysis (edited by S.P. Singh and J.H. Burry), Marcel Dekker, Inc., New York 80 (1982), 179-189.

[5] Hans, O., Reduzierende zufällige transformationen, Czech. Math. J. 7 (1957), 154-158.

[6] Himmelberg, C.J., Measurable relations, Fund. Math. 87 (1975), 53-72.

[7] Itoh, S., A random fixed point theorem for a multivalued contraction mapping, Pacific J. Math. 68 (1977), 85-90.

[8] Itoh, S., Random fixed point theorems with an application to random differential equations in Banach spaces, J. Math. Anal. Appl. 67 (1979), 261-273.

[9] Kuratowski, K., Ryll-Nardzewski, C., A general theorem on selectors, Bull. Acad. 
Polon. Sci. Ser. Sci. Math. Astronom. Phys. 13 (1965), 397-403.

[10] Lin, T.C. Random approximations and random fixed point theorems for nonself maps, Proc. Amer. Math. Soc. 103 (1988), 1129-1135.

[11] Papageorgiou, N.S., Random fixed point theorems for measurable multifunctions in Banach spaces, Proc. Amer. Math. Soc. 97 (1986), 507-514.

[12] Sehgal, V.M., Singh, S.P., On random approximations and a random fixed point theorem for set-valued mappings, Proc. Amer. Math. Soc. 95 (1985), 91-94.

[13] Sehgal, V.M., Waters, C., Some random fixed point theorems for condensing operators, Proc. Amer. Math. Soc. 90 (1984), 425-429.

[14] Takahashi, W., A convexity in metric space and nonexpansive mappings I, Kodai Math. Sem. Rep. 22 (1970), 142-149. 


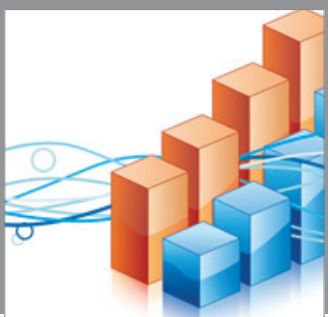

Advances in

Operations Research

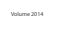

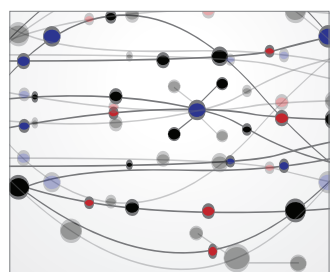

\section{The Scientific} World Journal
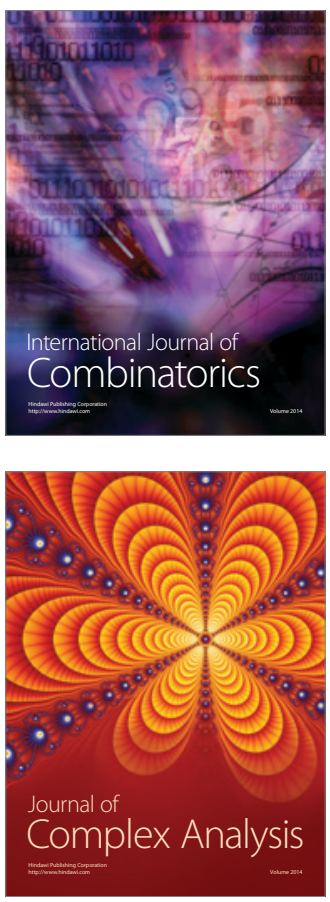

International Journal of

Mathematics and

Mathematical

Sciences
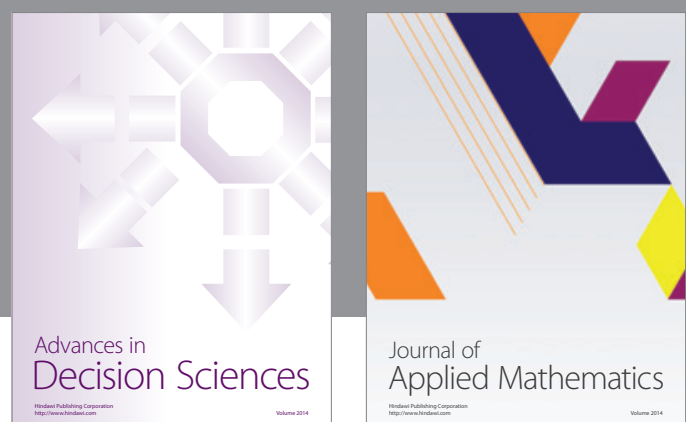

Journal of

Applied Mathematics
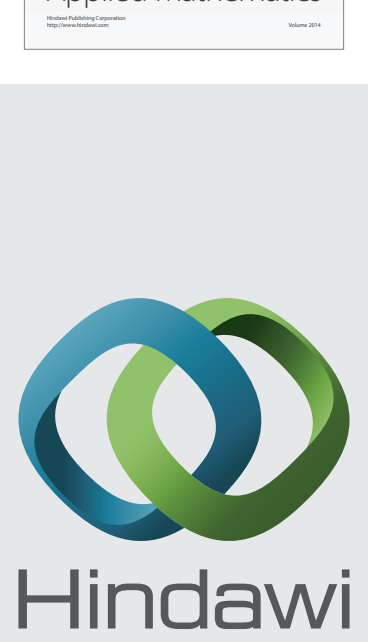

Submit your manuscripts at http://www.hindawi.com
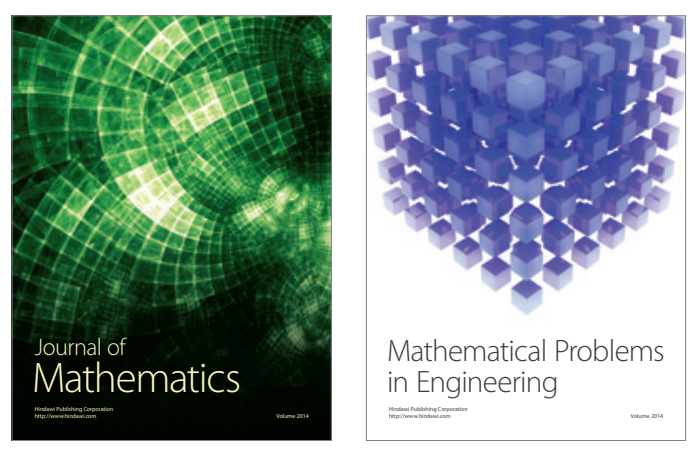

Mathematical Problems in Engineering
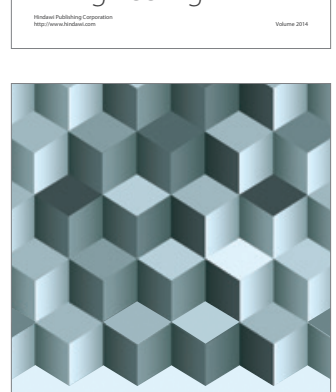

Journal of

Function Spaces
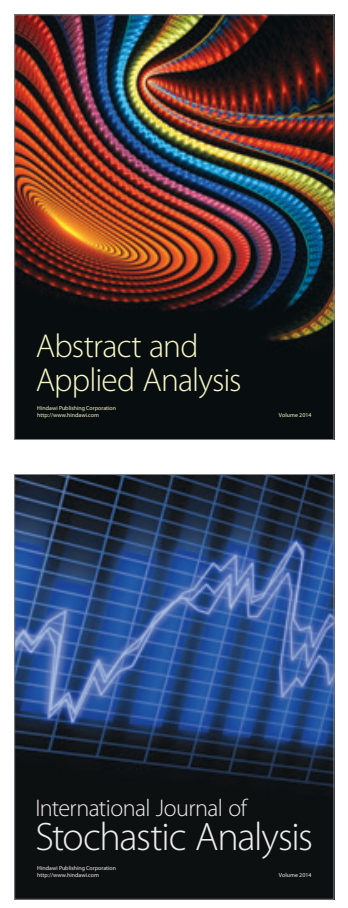

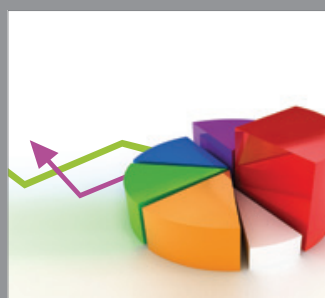

ournal of

Probability and Statistics

Promensencen
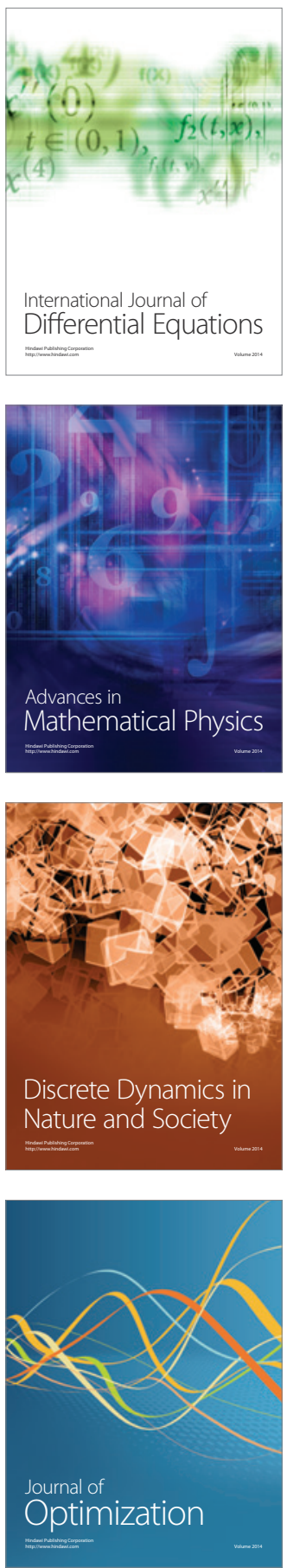